\title{
Improvement of the fire behavior of poly(1,4-butanediol succinate)/flax biocomposites by fiber surface modification with phosphorus compounds: molecular versus macromolecular strategy
}

\author{
Gaëlle Dorez, Belkacem Otazaghine, ${ }^{*}$ Aurélie Taguet, Laurent Ferry \\ and José-Marie Lopez-Cuesta
}

\begin{abstract}
The grafting of phosphorus compounds onto natural fibers has been investigated as a strategy for improving poly(1,4butanediol succinate)/flax biocomposite fire behavior. Three phosphorus compounds - dihydrogen ammonium phosphate, poly(methacryloyloxy)methyl phosphonic acid homopolymer and poly(methacryloyloxy)methyl phosphonic acid methylmethacrylate copolymer - were selected. The aim of this work was to compare the fire performance conferred by the grafted compounds depending on whether phosphorus is brought by a molecule or a macromolecule. TGA, pyrolysis combustion flow calorimetry and cone calorimetry were used to characterize the thermal stability and fire behavior of the samples. The pyrolysis combustion flow calorimetry results showed that in all cases the presence of phosphorus changes the degradation pathway and thus the flammability properties of flax. The ability of the grafted flame retardant to promote char formation and residue formation was found to be dependent on the nature and quantity of phosphorus covalently bonded to flax. Conversely, cone calorimeter tests revealed similar fire behavior whatever the grafting agent. A significant increase of the char amount and a global enhancement of fire parameters were observed with increasing grafting rate. Moreover, phosphonated polymers promoted a charred sheath around the fibers which acts in addition to their charring, conferring a fire performance close to that of dihydrogen ammonium phosphate for the biocomposite.
\end{abstract}

Keywords: biocomposite; natural fibers; fire behavior; phosphorus compound; molecule grafting; polymer grafting

\section{INTRODUCTION}

Natural fibers are emerging as prominent materials in producing ecofriendly composites. The considerable demands for natural fibers in the composite industry are due to their low cost, renewability, biodegradability, low density and good mechanical properties. However, their hydrophilic properties cause a weak interfacial adhesion between the fibers and polymeric matrix in reinforced composites. ${ }^{1-4}$ Moreover, the thermal and fire sensitivity of the fibers could limit their applications. ${ }^{5-7}$ An adapted treatment of the fibers could solve both problems.

Many papers are devoted to the development of flame retardants for cellulosic textiles or materials. ${ }^{8-10}$ Most of them are dedicated to organophosphorus treatments, e.g. THPC (tetrakis(hydroxymethyl)phosphonium chloride), Pyrovatex CP (N-methylol-3-(dimethylphosphono)propionamide) from Ciba-Geigy, SPDPC (spirocyclic penthaerythritol di(phosphonyl chloride)), APP (ammonium polyphosphate), DAP (dihydrogen ammonium phosphate), PA (phosphoric acid), TBP (tributyl phosphate), TAP (triallyl phosphate), TPT (triallyl phosphoric triamide) etc. Gaan et al. ${ }^{8}$ compared the effect of six organophosphorus compounds on the flame retardancy of cotton cellulose (Pyrovatex
CP, DAP, PA, TBP, TAP, TPT). They identified that Pyrovatex CP, DAP and $P A$ are more efficient than the other three. These three fire retardant agents maintain the integrity of the fiber after burning and allow the formation of a layer which insulates the cellulosic fiber. The three phosphorus compounds have a high activation energy of decomposition which leads to a higher char residue and a lower heat of combustion. ${ }^{8}$ Horrocks et al. ${ }^{11}$ have studied the substitution of the active hydrogens of cellulose by SPDPC. The phosphorylation of the cellulose allows the formation of a char due to the presence of penthaerythritol (which is a carbon source) in SPDPC. They identified that the enhancement of the char residue was linear between $0.5 \%$ and $2.5 \%$ of phosphorus; after this concentration, no significant effect was observed.

A limited number of papers deal with the fire retardancy of biocomposites by phosphorus grafting on natural fibers. ${ }^{12-16}$ Shumao et al. ${ }^{12}$ have demonstrated the efficiency of phosphorus

\footnotetext{
* Correspondence to: Belkacem Otazaghine, C2MA, Ecole des Mines d'Alès, 30319 Alès, France. E-mail: belkacem.otazaghine@mines-ales.fr
}

C2MA, Ecole des Mines d'Alès, 30319 Alès, France 


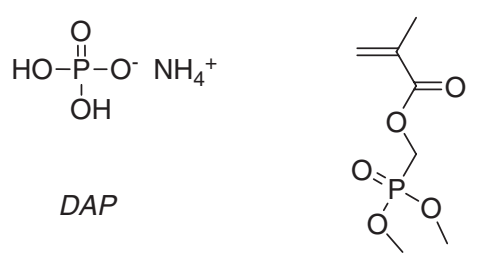

$M A P C_{1}$<smiles>CCC(C)(C)C(=O)OCP(=O)(O)O</smiles>

$P\left(M_{A P C}(\mathrm{OH})_{2}\right)$

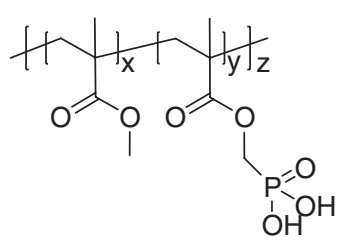

$P\left(M M A-C O-M A P C_{1}(\mathrm{OH})_{2}\right)$

Figure 1. Chemical formulae of DAP, $M A P C_{1}, P\left(M A P C_{1}(O H)_{2}\right)$ and $P\left(M M A-C O-M A P C_{1}(O H)_{2}\right)$.

(a) Synthesis of hydrolyzed MAPC1 homopolymer<smiles>C=C(C)C(=O)OCP(=O)(OC)OC</smiles><smiles>COP(=O)(COC(=O)C(C)(C)C)OCC(=O)C(C)(C)CC(C)(C)C</smiles>

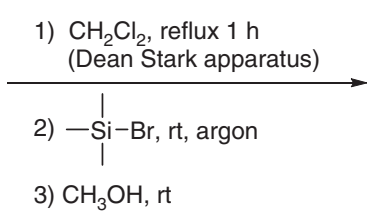<smiles>CC(C)(C)C(C(=O)OCP(=O)(O)O)C(C)(C)C</smiles>

(b) Synthesis of hydrolyzed MMA/MAPC1 copolymer<smiles>C=C(C)C(=O)OCP(=O)(OC)OC</smiles>

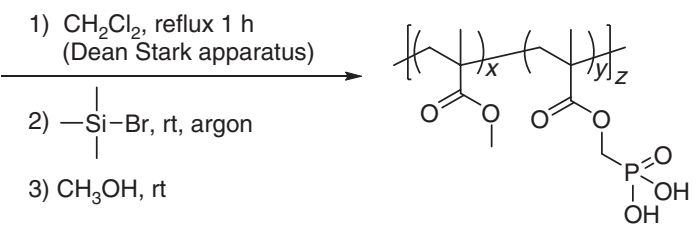

Figure 2. Synthesis of (a) homopolymer $\mathrm{P}\left(\mathrm{MAPC}_{1}(\mathrm{OH})_{2}\right)$ and (b) copolymer $\mathrm{P}\left(\mathrm{MMA}-\mathrm{co}-\mathrm{MAPC}_{1}(\mathrm{OH})_{2}\right)$.

surface treatment, because of the targeted action of the phosphorus compound (in this case APP) on ramie fibers. This action limits the heat and mass transfer from the polymer to the flame and improves the fire behavior of the poly(lactic acid) (PLA)/ramie biocomposite. However, to obtain satisfactory results in the UL94 rating, a combined use of APP in the matrix and on the natural fiber is required. Chen et al. ${ }^{13}$ also tried to improve the fire behavior of PLA/ramie with APP and with microencapsulated APP (MAPP) as a fire retardant treatment. They investigated the fire behavior of biocomposites after UV irradiation and hydrothermal aging. The use of MAPP deposited onto the natural fibers and added in the PLA matrix gives the best result in UL94 rating (only decreasing from V0 to V1 after 21 days' exposure). This rating is due to the good dispersion of MAPP in the matrix and to the good interaction between MAPP and ramie fiber. Suardana et al. ${ }^{14}$ have worked on biocomposites based on PLA and polypropylene (PP) as the matrix, coconut and jute as the natural fibers and DAP as the treatment agent. They showed that an increase of the DAP grafting rate on natural fibers leads to a decrease of the burning rate and the weight loss.

In a previous study, we have demonstrated that a covalent bond is formed between the flax fiber and the phosphonic acid function in soft conditions. ${ }^{16}$ The presence of the phosphorus compound (DAP or PA) grafted onto the fiber presents two main advantages compared with fireproofing by incorporation into the matrix: no hydrolysis of poly(1,4-butanediol succinate) (PBS) and a good fire behavior with low phosphorus content (around $1 \mathrm{wt} \%) .{ }^{15}$ A better understanding of the interaction between phosphorus compounds and natural fibers could lead to improvement in the thermal and fire behavior of biocomposites.

In the present work, we decided to investigate the improvement of the fire behavior of PBS/flax biocomposites by grafting of phosphorus compound on flax fibers. We compared different fire retardant treatment agents: a phosphonated molecule (DAP) and two phosphonated polymers - a homopolymer of ((methacryloyloxy)methyl)phosphonic acid $\left(\mathrm{P}\left(\mathrm{MAPC}_{1}(\mathrm{OH})_{2}\right)\right)$ and a copolymer of this monomer with methylmethacrylate (P(MMACo-MAPC $\left.(\mathrm{OH})_{2}\right)$ ). The objective of this study was to determine the impact of the structure of phosphorus compounds on the thermal and fire properties of flax fibers and PBS composites.

\section{EXPERIMENTAL}

\section{Materials}

PBS (film grade 1903 F, from Xinfu Pharm, China) was used as the matrix. Flax fibers (Fibras-S6B) were produced in France and were supplied from FRD ${ }^{\circledR}$. Cellulose, hemicelluloses and lignin are the main components of these fibers (80, 13 and 2 wt\% respectively). Dimethyl(methacryloxy)methyl phosphonate $\left(\mathrm{MAPC}_{1}\right)$ (Specific Polymer, France), methylmethacrylate (Sigma Aldrich, France), bromotrimethylsilane (Sigma Aldrich), 2-2'-azobis(2-methylpropionitrile) at 98\% (AIBN) (Sigma Aldrich) and DAP (Prolabo, France) were used as received. The chemical structures of DAP and MAPC 1 are given in Fig. 1.

\section{Synthesis of phosphonated polymers}

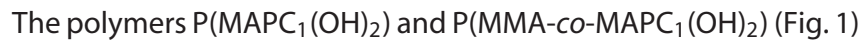
were prepared by a two-step synthesis described in Fig. 2.

\section{Synthesis of $\mathrm{P}\left(\mathrm{MMA}-\mathrm{co}-\mathrm{MAPC}_{\mathbf{1}}(\mathrm{OH})_{2}\right)$ (50/50 mol\%)}

The first step consisted in a radical copolymerization of MMA and MAPC $_{1}$ under an argon atmosphere. Into a $100 \mathrm{~mL}$ flask fitted with a condenser $7.2 \mathrm{~g}\left(7.2 \times 10^{-2} \mathrm{~mol}\right)$ of MMA, $15 \mathrm{~g}\left(7.2 \times 10^{-2} \mathrm{~mol}\right)$ 


\begin{tabular}{|c|c|c|c|c|c|c|}
\hline & Designation & $\begin{array}{l}\text { PBS } \\
\text { (wt\%) }\end{array}$ & $\begin{array}{l}\text { DAP } \\
(w t \%)\end{array}$ & $\begin{array}{c}\mathrm{P}\left(\mathrm{MAPC}_{1}(\mathrm{OH})_{2}\right) \\
(\mathrm{wt} \%)\end{array}$ & $\begin{array}{c}\mathrm{P}(\mathrm{MMA}-\mathrm{co}- \\
\left.\mathrm{MAPC}_{1}(\mathrm{OH})_{2}\right)(\mathrm{wt} \%)\end{array}$ & $\begin{array}{c}\text { Tfl } \\
\text { (wt\%) }\end{array}$ \\
\hline \multirow[t]{3}{*}{ FTfl } & Tfl- $x$ DAP & & $x$ & & & $100-x$ \\
\hline & Tfl-yP(MAPC $\left.{ }_{1}(\mathrm{OH})_{2}\right)$ & & & $y$ & & $100-y$ \\
\hline & Tfl-zP(MMA-co-MAPC $\left.1(\mathrm{OH})_{2}\right)$ & & & & $z$ & $100-z$ \\
\hline \multirow[t]{2}{*}{ PBS-Tfl } & PBS+Tfl & 70 & & & & 30 \\
\hline & PBS+Tfl-xDAP & 70 & $30 x$ & & & $30(1-x)$ \\
\hline \multirow[t]{2}{*}{ PBS-FTfl } & $\mathrm{PBS}+\mathrm{Tfl}-\mathrm{yP}\left(\mathrm{MAPC}_{1}(\mathrm{OH})_{2}\right)$ & 70 & & $30 y$ & & $30(1-y)$ \\
\hline & $\mathrm{PBS}+\mathrm{Tfl}-z \mathrm{P}\left(\mathrm{MMA}-\mathrm{Co}-\mathrm{MAPC}_{1}(\mathrm{OH})_{2}\right)$ & 70 & & & $30 z$ & $30(1-z)$ \\
\hline
\end{tabular}
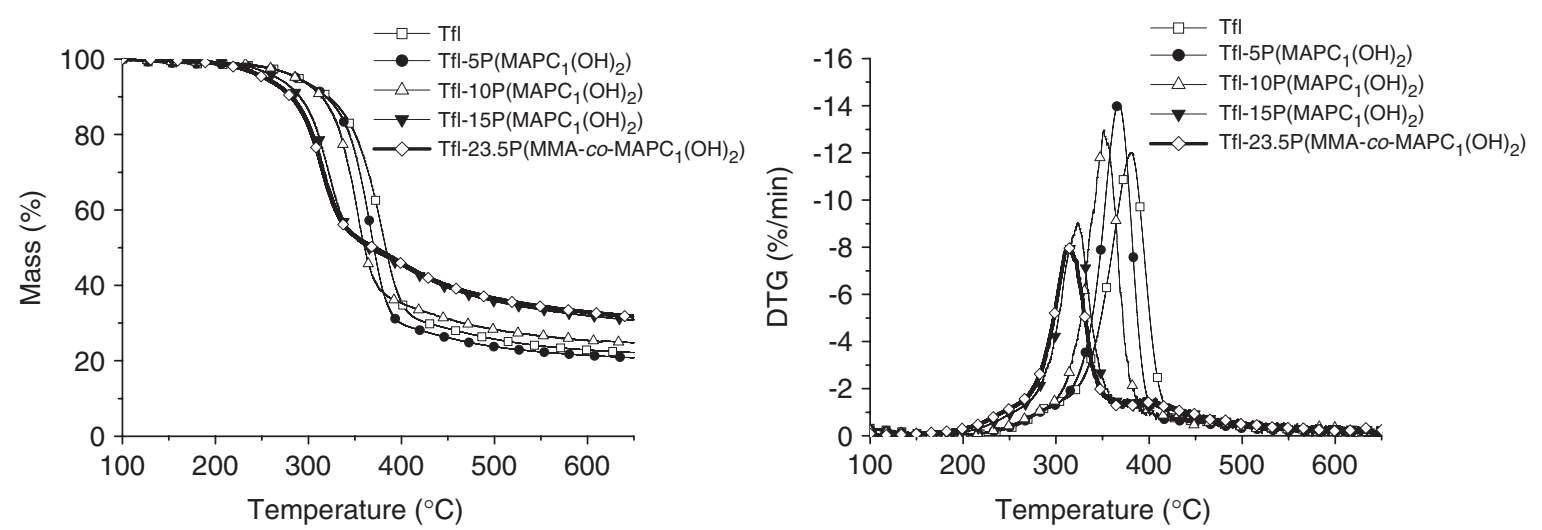

Figure 3. TGA curves of Tfl, Tfl-yP(MAPC $\left.(\mathrm{OH})_{2}\right)$ and Tfl-23.5P(MMA-co-MAPC $\left.{ }_{1}(\mathrm{OH})_{2}\right)$.

of MAPC $_{1}, 0.24 \mathrm{~g}\left(1.5 \times 10^{-3} \mathrm{~mol}\right)$ of AIBN and $22 \mathrm{~g}$ of acetonitrile were introduced. Argon was bubbled through the mixture for 15 min. The mixture was then stirred and heated at $80^{\circ} \mathrm{C}$ for $15 \mathrm{~h}$. After reaction the polymer was purified by precipitation in diethylether.

The second step consisted in hydrolysis of the phosphonate groups of the copolymer obtained from the first step. Into a 100 $\mathrm{mL}$ two-necked flask equipped with a Dean Stark apparatus, 10 $\mathrm{g}$ of copolymer $\mathrm{P}\left(\mathrm{MMA}^{\mathrm{N}} \mathrm{-CO}-\mathrm{MAPC}_{1}\right)(50 / 50 \mathrm{~mol} \%)$ and $40 \mathrm{~mL}$ of dichloromethane were introduced, under an inert atmosphere. The mixture was then degassed by bubbling argon for 15 min, stirred and heated at dichloromethane reflux for $1 \mathrm{~h}$ (water was eliminated by the Dean Stark apparatus). The mixture was then cooled at room temperature and $10.6 \mathrm{~g}\left(6.9 \times 10^{-2} \mathrm{~mol}\right)$ of bromotrimethylsilane was added dropwise. After $15 \mathrm{~h}$ the solvent was eliminated under vacuum and $80 \mathrm{~mL}$ of methanol was added. Finally, the mixture was stirred for $3 \mathrm{~h}$ before precipitation of the copolymer $\mathrm{P}\left(\mathrm{MMA}-\mathrm{CO}_{\mathrm{MAPC}}(\mathrm{OH})_{2}\right)$ in diethylether. ${ }^{17-19}$

The same procedure was used to synthesize the homopolymer $\mathrm{P}\left(\mathrm{MAPC}_{1}(\mathrm{OH})_{2}\right)$.

\section{Fiber treatment}

Pretreatment of fibers

First, the flax fibers were pretreated with ethanol to remove waxes and pectin, which ensure the cohesion of the flax beam. ${ }^{20}$ Into a $1 \mathrm{~L}$ reactor equipped with a condenser and a mechanical stirrer, $50 \mathrm{~g}$ of flax fibers and $800 \mathrm{~mL}$ of ethanol were introduced. The mixture was stirred and heated under reflux for $5 \mathrm{~h}$. The liquid phase was then eliminated by filtration and the flax fibers were washed three times with ethanol and dried at room temperature. The fiber samples obtained by this treatment were noted Tfl.

\section{Grafting procedure of fibers}

The procedure used for the grafting of the flax fibers is very similar to that for the fiber pretreatment. Into a $1 \mathrm{~L}$ reactor equipped with a condenser and a mechanical stirrer, $45 \mathrm{~g}$ of Tfl, $5 \mathrm{~g}$ of treatment agent (for a $10 \mathrm{wt} \%$ treatment) and $800 \mathrm{~mL}$ of ethanol (96\%) were introduced. The mixture was stirred and heated under reflux for 5 h. The liquid phase was then eliminated by filtration and the flax fibers were washed three times with ethanol and dried at room temperature. The modified fiber samples were noted FTfl (Table 1).

For the treatment with DAP, flax fibers were washed three times after reaction and dried at room temperature. For $\mathrm{P}\left(\mathrm{MAPC}_{1}(\mathrm{OH})_{2}\right)$ and $\mathrm{P}\left(\mathrm{MMA}-\mathrm{CO}_{\mathrm{MAPC}}(\mathrm{OH})_{2}\right)$ treatments, the solvent was eliminated under vacuum after reaction and the fibers were not washed. Indeed it was noted that after cooling at room temperature the reaction mixture became cloudy. It was assumed that this phenomenon was related to the solubilization of components of fibers (e.g. lignin) by hot ethanol after grafting of the phosphonated polymers. At room temperature the solubility of these complexes decreases and this could explain the hazy mixture.

\section{Composite preparation}

Blending was performed in a Haake Rheomix internal mixer (100 rpm at $110^{\circ} \mathrm{C}$ ). The PBS pellets were first introduced in the chamber and mixed for $2.5 \mathrm{~min}$ until a constant torque was reached. Then, pretreated (Tfl) or treated (FTfl) fibers were introduced progressively during $9 \mathrm{~min}$. After the final fiber addition, the mixing was extended for $2 \mathrm{~min}$. All materials were compression molded at 110 bar into $100 \times 100 \times 4 \mathrm{~mm}^{3}$ sheets weighing around $50 \mathrm{~g}$. Table 1 shows the compositions of the different biocomposites. 


\begin{tabular}{|c|c|c|c|c|c|c|c|}
\hline \multirow[b]{2}{*}{ Sample } & \multicolumn{2}{|c|}{ TGA } & \multicolumn{4}{|c|}{ PCFC } & \multirow[b]{2}{*}{$\% \mathrm{P}^{\mathrm{a}}$} \\
\hline & $T_{\text {deg }}\left({ }^{\circ} \mathrm{C}\right)$ & $\operatorname{Res}_{600}(\%)$ & $T_{\text {deg }}\left({ }^{\circ} \mathrm{C}\right)$ & $\mathrm{pHRR}\left(\mathrm{kW} \mathrm{m}^{-2}\right)$ & $\operatorname{THR}\left(\mathrm{kJ} \mathrm{g}^{-1}\right)$ & $\mathrm{EHC}\left(\mathrm{kJ} \mathrm{g}^{-1}\right)$ & \\
\hline Tfl & 380.6 & 22.9 & 370.7 & 131.8 & 7.1 & 9.2 & - \\
\hline Tfl-2.5DAP & 335.3 & 29.4 & 326.0 & 57.4 & 7.8 & 11.0 & 0.39 \\
\hline Tfl-5DAP & 321.1 & 31.3 & 318.4 & 118.9 & 5.7 & 8.3 & 0.55 \\
\hline Tfl-7.5DAP & 297.6 & 40.2 & 298.6 & 76.8 & 3.6 & 6.0 & 0.90 \\
\hline Tfl-10DAP & 303.2 & 40.5 & 299.6 & 50.1 & 2.7 & 4.5 & 1.13 \\
\hline Tfl-5P(MAPC $\left.{ }_{1}(\mathrm{OH})_{2}\right)$ & 367.1 & 21.5 & 361.2 & 149.7 & 8.0 & 10.2 & 0.85 \\
\hline Tfl-10P(MAPC $\left.{ }_{1}(\mathrm{OH})_{2}\right)$ & 355.3 & 25.3 & 345.7 & 120.8 & 8.1 & 10.8 & 1.7 \\
\hline Tfl-15P(MAPC $\left.1(\mathrm{OH})_{2}\right)$ & 323.2 & 32.0 & 332.5 & 88.0 & 5.9 & 8.7 & 2.55 \\
\hline Tfl-23.5P(MMA-co-MAPC $\left.1(\mathrm{OH})_{2}\right)$ & 312.1 & 31.6 & 318.0 & 87.9 & 5.8 & 8.5 & 2.55 \\
\hline
\end{tabular}

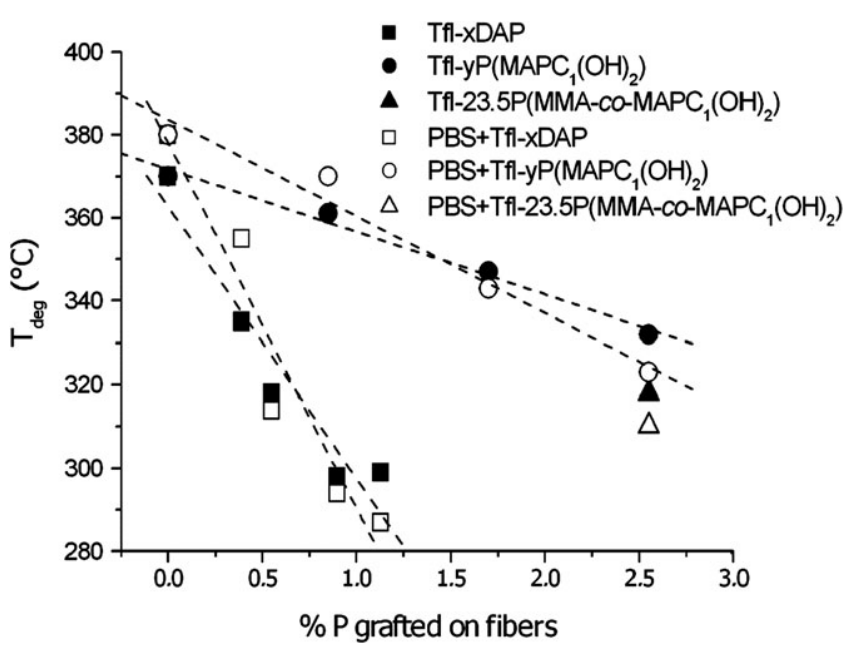

Figure 4. Degradation temperature for FTfl (full symbols) and first peak of degradation for PBS-FTf (empty symbols) versus percentage of phosphorus grafted on flax.

\section{Characterization}

Thermogravimetric analysis

TGA for the treated fibers and biocomposites was carried out on a Perkin Elmer Pyris-1 Thermogravimetric Analyzer instrument. Sample weights of around $10 \mathrm{mg}$ were heated from 50 to $900^{\circ} \mathrm{C}$ at a heating rate of $10{ }^{\circ} \mathrm{C} \mathrm{min}^{-1}$ under a nitrogen atmosphere. Values of the residual weight at $600{ }^{\circ} \mathrm{C}\left(\operatorname{Res}_{600}\right)$ and the temperature of maximum degradation ( $\left.T_{\text {deg }}\right)$ were determined for each sample.

\section{Pyrolysis combustion flow calorimetry}

Flammability properties were obtained by pyrolysis combustion flow calorimetry (PCFC). This technique has been developed by Lyon and Walters. ${ }^{21}$ Sample weights of around $2-5 \mathrm{mg}$ were pyrolyzed at $1{ }^{\circ} \mathrm{C} \mathrm{s}^{-1}$ and the degradation products were introduced in a combustor at $900{ }^{\circ} \mathrm{C}$, where they were completely oxidized. The peak heat release rate (pHRR), temperature of degradation $\left(T_{\text {deg }}\right)$, total heat release (THR) and effective heat of combustion $(\mathrm{EHC})$ were determined.

\section{Cone calorimetry}

A Fire Testing Technology cone calorimeter was used to evaluate fire reaction properties. Samples of $100 \times 100 \times 4 \mathrm{~mm}^{3}$ were

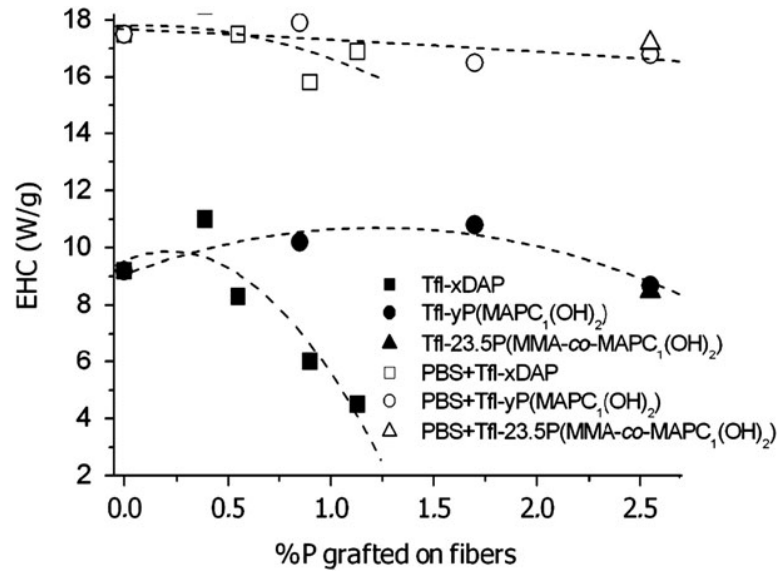

Figure 5. Effective heat of combustion of FTfl (full symbols) and PBS-FTfl (empty symbols) versus percentage of phosphorus grafted on flax.

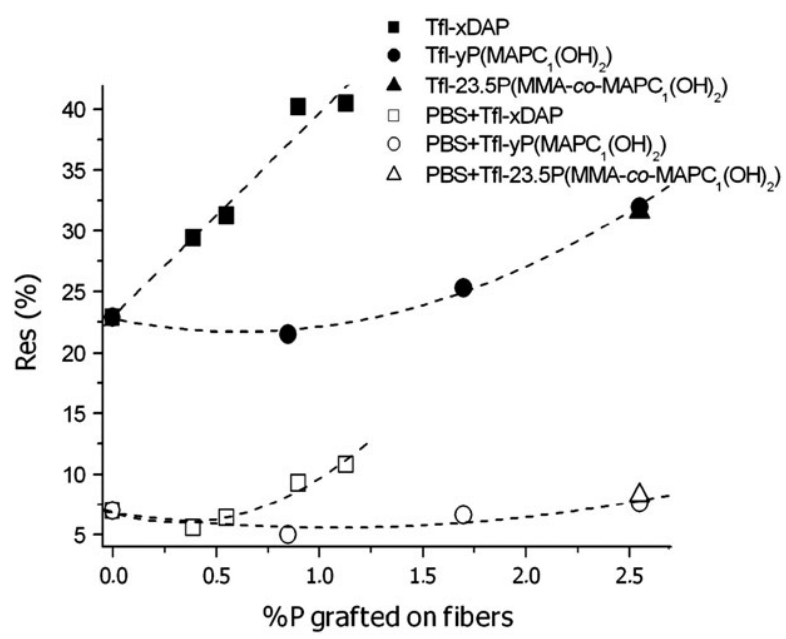

Figure 6. Percentage of residue for FTfl (full symbols) and PBS-FTfl (empty symbols) samples versus percentage of phosphorus grafted on flax.

exposed to a $35 \mathrm{~kW} \mathrm{~m}^{-2}$ radiant heat flux. This irradiance corresponds to a common heat flux in a developing fire scenario. The time to ignition (TTI), pHRR, THR, EHC, char residue $\left(X_{\text {char }}\right)$ and maximum average of heat emission (MARHE) were determined. 
Char due to the phosphorylation of the fiber
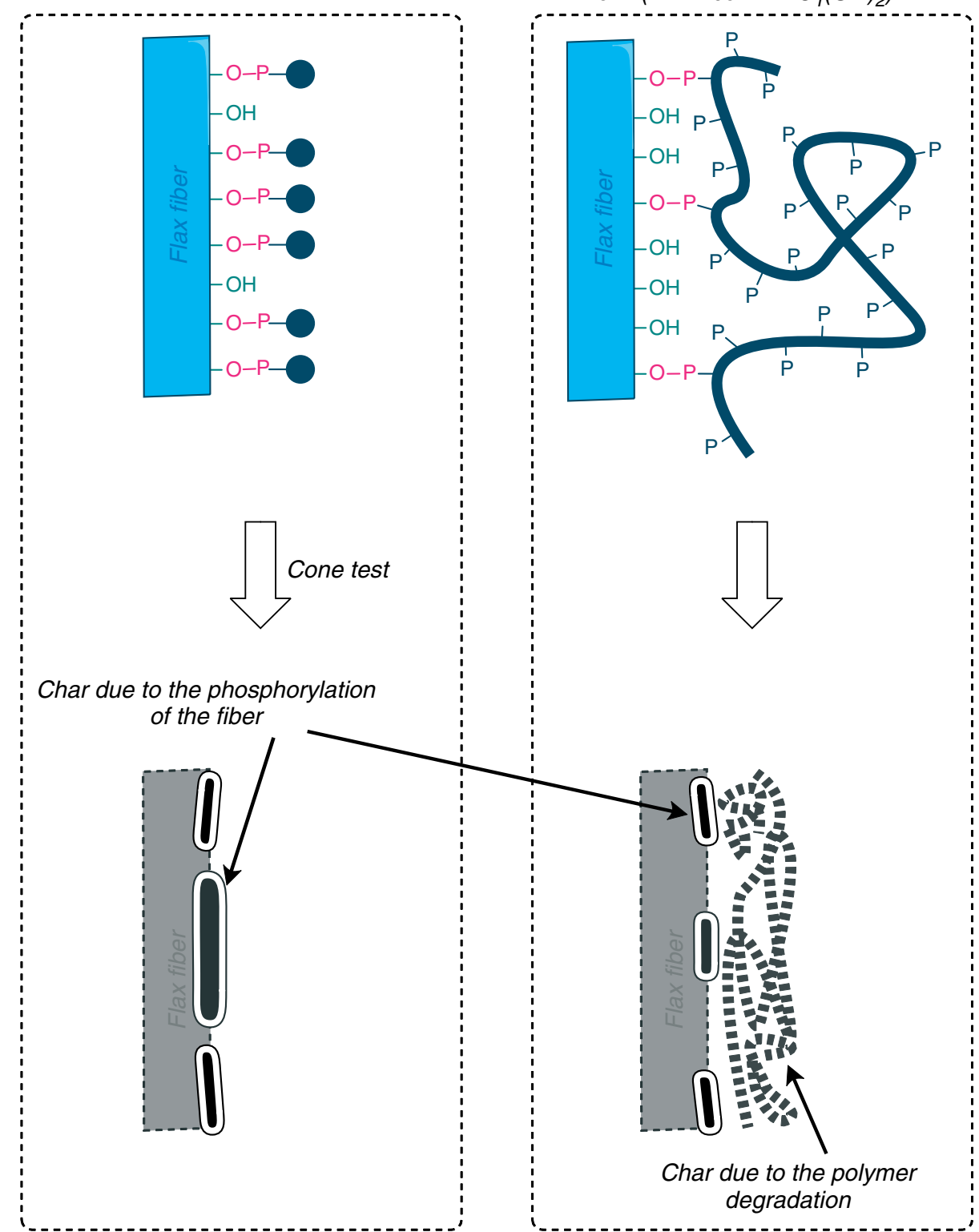

Fiber modified with $\mathrm{P}\left(\mathrm{MAPC} \mathrm{C}_{1}(\mathrm{OH})_{2}\right)$ or $P\left(M M A-c o-M A P C_{1}(O H)_{2}\right)$

Figure 7. Scheme of the grafting of molecules (DAP) and polymers on flax fibers before and after the cone calorimeter test.

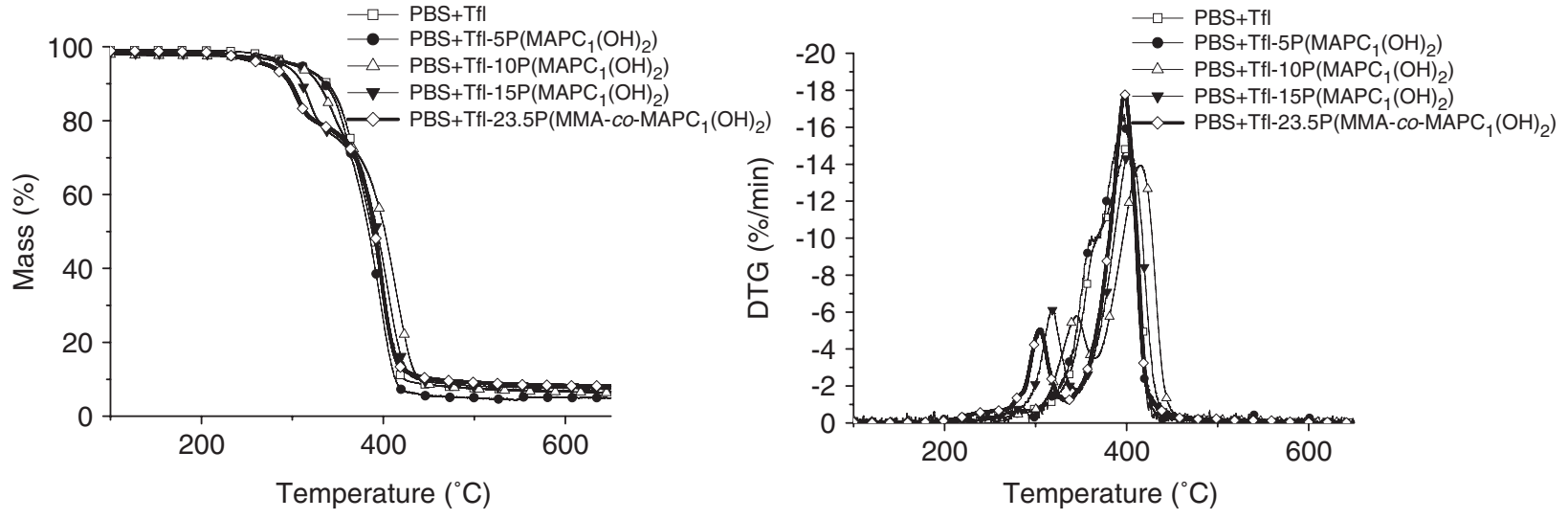

Figure 8. TGA curves of PBS+Tfl, $\mathrm{PBS}+\mathrm{Tfl}-\mathrm{P} P\left(\mathrm{MAPC}_{1}(\mathrm{OH})_{2}\right)$ and $\mathrm{PBS}+\mathrm{Tfl}-23.5 \mathrm{P}\left(\mathrm{MMA}-\mathrm{CO}-\mathrm{MAPC}_{1}(\mathrm{OH})_{2}\right)$. 
Table 3. TGA and PCFC parameters of PBS+Tfl and PBS+FTfl

\begin{tabular}{|c|c|c|c|c|c|c|}
\hline \multirow[b]{2}{*}{ Samples } & \multicolumn{2}{|c|}{ ATG } & \multicolumn{4}{|c|}{ PCFC } \\
\hline & $T_{\text {deg }}\left({ }^{\circ} \mathrm{C}\right)$ & $\operatorname{Res}_{600}(\%)$ & $T_{\text {deg }}\left({ }^{\circ} \mathrm{C}\right)$ & $\mathrm{pHRR}\left(\mathrm{kW} \mathrm{m}^{-2}\right)$ & $\operatorname{THR}\left(\mathrm{kJ} \mathrm{g}^{-1}\right)$ & $\mathrm{EHC}\left(\mathrm{kJ} \mathrm{g}^{-1}\right)$ \\
\hline PBS+Tfl & 401.2 & 7.0 & $393.3 / 421.8$ & $126.3 / 264.7$ & 16.3 & 17.5 \\
\hline PBS+Tfl-2.5DAP & 400.0 & 5.6 & $355.2 / 419.0$ & $56.6 / 296.8$ & 17.2 & 18.5 \\
\hline PBS+Tfl-5DAP & $319.5 / 404.5$ & 6.4 & $314.2 / 422.0$ & $59.1 / 280.9$ & 16.4 & 17.5 \\
\hline PBS+Tfl-7.5DAP & $288.1 / 400.9$ & 9.3 & $294.1 / 418.8$ & $47.0 / 271.0$ & 15.3 & 15.8 \\
\hline PBS+Tfl-10DAP & $284.5 / 399.3$ & 10.8 & $286.8 / 413.4$ & $49.5 / 300.3$ & 15.1 & 16.9 \\
\hline $\mathrm{PBS}+\mathrm{Tfl}-5 \mathrm{P}\left(\mathrm{MAPC}_{1}(\mathrm{OH})_{2}\right)$ & $361.5 / 397.4$ & 5.0 & $383 / 416.2$ & $99.8 / 240.3$ & 16.7 & 17.9 \\
\hline $\mathrm{PBS}+\mathrm{Tfl}-10 \mathrm{P}\left(\mathrm{MAPC}_{1}(\mathrm{OH})_{2}\right)$ & $344.2 / 413.3$ & 6.6 & $343 / 414.0$ & $65.7 / 268.1$ & 15.7 & 16.5 \\
\hline $\mathrm{PBS}+\mathrm{Tfl}-15 \mathrm{P}\left(\mathrm{MAPC}_{1}(\mathrm{OH})_{2}\right)$ & $318.7 / 406.7$ & 7.6 & $323.8 / 418.7$ & $67.9 / 275.4$ & 15.5 & 16.8 \\
\hline PBS+Tfl-23.5P(MMA-co-MAPC $\left.{ }_{1}(\mathrm{OH})_{2}\right)$ & $304.9 / 398.7$ & 8.3 & $310.2 / 414.6$ & $50.8 / 316.2$ & 15.8 & 17.2 \\
\hline
\end{tabular}
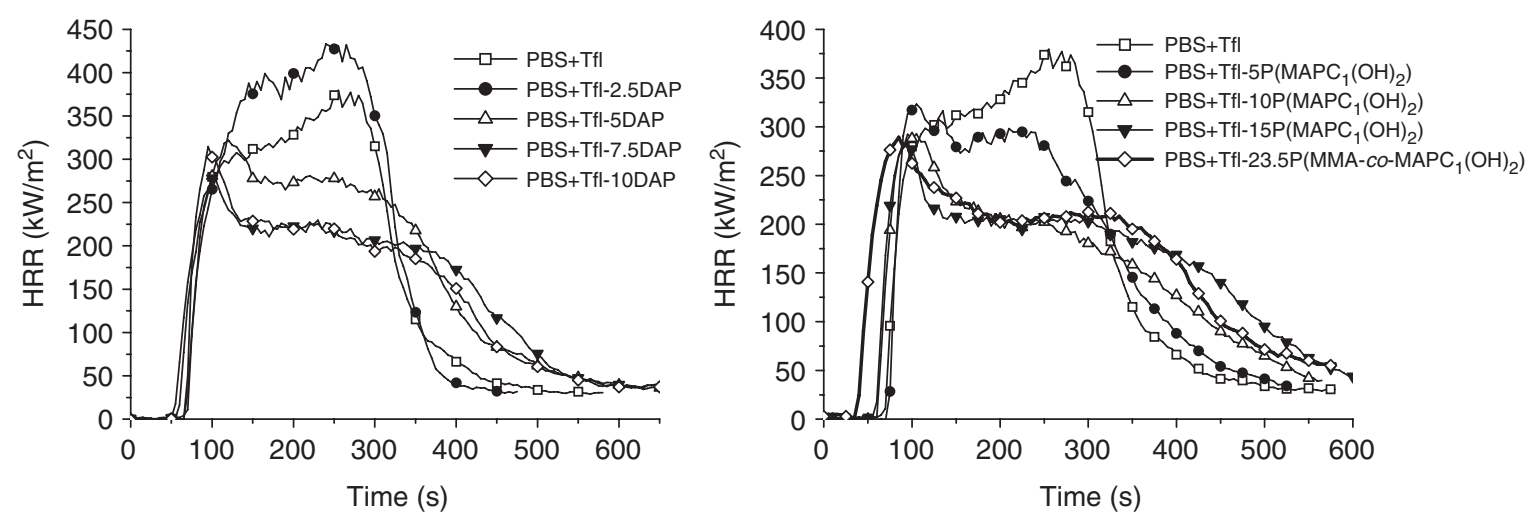

Figure 9. Cone calorimeter curves of PBS-FTfl.

The ARHE was calculated according to ${ }^{22}$

$$
\operatorname{ARHE}\left(t_{n}\right)=\frac{\sum_{2}^{n}\left(t_{n}-t_{n-1}\right)\left(q_{n}+q_{n-1}\right) / 2}{t_{n}-t_{0}}
$$

where $t_{n}$ is the time and $q_{n}$ is the rate of heat released at $t_{n}$.

\section{Elemental analysis}

For phosphorus elemental analysis, the samples were dissolved in an acid solution and analyzed by inductively coupled plasma atom emission spectroscopy. Elemental analysis allows the initial phosphorus content of DAP treated fibers to be measured. For the $\mathrm{P}\left(\mathrm{MAPC}_{1}(\mathrm{OH})_{2}\right)$ and $\mathrm{P}\left(\mathrm{MMA}-\mathrm{CO}-\mathrm{MAPC}_{1}(\mathrm{OH})_{2}\right)$ treated fibers, the phosphorus content was deduced from the phosphorus content of the considered polymer, since all the treatment agent was supposed to be deposited onto the fibers.

\section{RESULTS AND DISCUSSION \\ Grafted flax fibers}

Different amounts of DAP $(2.5,5,7.5$ and $10 \mathrm{wt} \%), \mathrm{P}\left(\mathrm{MAPC}_{1}(\mathrm{OH})_{2}\right)$ $(5,10$ and 15 wt $\%)$ and $\mathrm{P}\left(\mathrm{MMA}-\mathrm{CO}-\mathrm{MAPC}_{1}(\mathrm{OH})_{2}\right)(23.5 \mathrm{wt} \%)$ were grafted onto pretreated flax fibers. In Fig. 3 only the TGA curves of flax treated with $\mathrm{P}\left(\mathrm{MAPC}_{1}(\mathrm{OH})_{2}\right)$ and $\mathrm{P}\left(\mathrm{MMA}-\mathrm{CO}-\mathrm{MAPC}_{1}(\mathrm{OH})_{2}\right)$ are presented; however, flax treated with DAP exhibits the same trend. ${ }^{15}$ The thermal degradation of the pretreated and grafted flax fibers occurs in one step, similarly to raw flax fibers. $^{6,23}$ The grafted fibers start to decompose earlier when

the grafting rate is increased. An increase of the residue, up to $77 \%$ for the Tfl-10DAP, $40 \%$ for the Tfl-15P( $\left(\mathrm{MAPC}_{1}(\mathrm{OH})_{2}\right)$ and $38 \%$ for the Tfl-23.5P(MMA-Co-MAPC1 $\left.(\mathrm{OH})_{2}\right)$, is observed. According to the literature, ${ }^{24,25}$ the decrease of the thermal stability and the significant increase in the amount of residue can be explained by a change in the decomposition pathway of cellulose (main part of the flax fibers). Cellulose can decompose either by dehydration with the formation of a char, $\mathrm{H}_{2} \mathrm{O}, \mathrm{CO}$ and $\mathrm{CO}_{2}$ or by depolymerization with the formation of tar and levoglucosane (a highly flammable compound). As explained by Lewin and Weil, ${ }^{26}$ the presence of phosphorus compounds leads to the phosphorylation of cellulose, due to the phosphoric acid released during decomposition of the fire retardant. This phosphorylation favors dehydration to the detriment of depolymerization and leads to a decrease of the thermal stability and to enhancement of char formation. ${ }^{26,27}$ In the present case, it must be emphasized that phosphorylation of cellulose was already achieved during fiber treatment.

Moreover, a decrease of the pHRR and EHC parameters with increasing grafting rate (except at low content of phosphorus) is highlighted in PCFC (Table 2). These differences can also be assigned to the change of the degradation pathway when phosphorus is grafted onto fibers.

To compare the effect of the two types of fire retardant agents, molecule (DAP) and polymer $\left(\mathrm{P}\left(\mathrm{MAPC}_{1}(\mathrm{OH})_{2}\right)\right.$ and $\mathrm{P}(\mathrm{MMA}-$ Co-MAPC $\left.(\mathrm{OH})_{2}\right)$ ), the temperature of the peak of degradation obtained in PCFC (Fig. 4), EHC (Fig. 5) and the residue (Fig. 6) of treated fibers (full symbols) are plotted as a function of phosphorus content. 


\begin{tabular}{|c|c|c|c|c|c|c|}
\hline Samples & TTI (s) & $\operatorname{pHRR}\left(\mathrm{kW} \mathrm{m}^{-2}\right)$ & $\operatorname{THR}\left(\mathrm{kJ} \mathrm{g}{ }^{-1}\right)$ & $\mathrm{EHC}\left(\mathrm{kJ} \mathrm{g}^{-1}\right)$ & Xchar (\%) & MARHE $\left(\mathrm{kW} \mathrm{m}^{-2}\right)$ \\
\hline PBS+Tfl & 68 & 379.5 & 17.7 & 18.4 & 4.2 & 241.7 \\
\hline PBS+Tfl-10DAP & 64 & 314.5 & 16.1 & 17.7 & 9.2 & 178.6 \\
\hline PBS+Tfl-7.5DAP & 50 & 278.2 & 16.1 & 17.5 & 8.2 & 181.8 \\
\hline PBS+Tfl-5DAP & 56 & 321.8 & 17.3 & 18.4 & 6.1 & 216.1 \\
\hline PBS+Tfl-2.5DAP & 63 & 433.0 & 18.0 & 18.9 & 4.5 & 277.5 \\
\hline $\mathrm{PBS}+\mathrm{Tfl}-5 \mathrm{P}\left(\mathrm{MAPC}_{1}(\mathrm{OH})_{2}\right)$ & 70 & 323.1 & 17.4 & 18.7 & 7.0 & 208.4 \\
\hline $\mathrm{PBS}+\mathrm{Tfl}-10 \mathrm{P}\left(\mathrm{MAPC}_{1}(\mathrm{OH})_{2}\right)$ & 57 & 292.6 & 16.6 & 18.3 & 9.4 & 172.0 \\
\hline $\mathrm{PBS}+\mathrm{Tfl}-15 \mathrm{P}\left(\mathrm{MAPC}_{1}(\mathrm{OH})_{2}\right)$ & 52 & 291.4 & 17.0 & 18.7 & 9.2 & 189.7 \\
\hline PBS+Tfl-23.5P(MMA-co-MAPC $\left.{ }_{1}(\mathrm{OH})_{2}\right)$ & 36 & 289.1 & 18.1 & 19.3 & 6.2 & 192.3 \\
\hline
\end{tabular}

It should be noted that, at equivalent phosphorus content, the decrease of the temperature of degradation is more pronounced

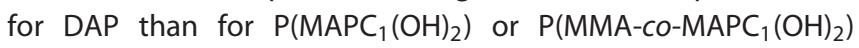
(Fig. 4). Furthermore, at equivalent phosphorus content, the two phosphonated polymers seem to induce a similar effect since $15 \mathrm{P}\left(\mathrm{MACP} \mathrm{P}_{1}(\mathrm{OH})_{2}\right)$ and 23.5P(MMA-CO-MAPC $\left.1(\mathrm{OH})_{2}\right)$ exhibit almost the same degradation temperature. If the temperature of degradation is assumed to be a good indicator of the degradation mechanism of cellulose, it can be concluded that DAP is more effective in switching the degradation pathway from depolymerization to dehydration.

From Fig. 5 it can be seen that the variation of EHC is significant when the DAP grafting rate increases, contrary to $\mathrm{P}\left(\mathrm{MAPC}_{1}(\mathrm{OH})_{2}\right)$ which exhibits low EHC variation. As previously mentioned, the fiber degradation pathway is more affected by DAP. Thus, the gases released during the degradation of Tfl-xDAP are to some extent different from those of unmodified fibers or Tfl- $x P\left(M A P C_{1}(O H)_{2}\right)$ and their oxidation is supposed to emit less energy.

The three fire retardant agents increase the decomposition residue of the fibers. However, the ability to form residue is more pronounced for DAP ( $40 \mathrm{wt} \%$ of residue at $1.13 \%$ of phosphorus for $\mathrm{DAP}$, and only $32 \mathrm{wt} \%$ at $2.55 \%$ of phosphorus for $\mathrm{P}\left(\mathrm{MAPC}_{1}(\mathrm{OH})_{2}\right)$ ). Once again the predominance of dehydration in the presence of DAP should be responsible for this discrepancy. Figures 5 and 6 show that the EHC is strongly correlated to the evolution of residue as a function of the percentage of phosphorus grafted. It appears that the released energy is very close for all compositions for a given mass loss.

The difference in structure between the phosphorus agents, as well as their grafting rate, leads to differences in the degradation pathway for the modified fibers. For either the molecule or the polymer, the phosphorylation of flax fibers favors their dehydration and enhances char formation. However, these actions are stronger with DAP. Hence, it is assumed that only a part of the phosphonic acid functions of each polymer chain is involved in the grafting of $\mathrm{P}\left(\mathrm{MAPC}_{1}(\mathrm{OH})_{2}\right)$ and $\mathrm{P}\left(\mathrm{MMA}-\mathrm{CO}-\mathrm{MAPC}_{1}(\mathrm{OH})_{2}\right)$ onto the fiber surface, contrary to DAP (Fig. 7). Thus it can be concluded that phosphorus covalently bonded to flax is more effective in favoring dehydration and increasing char yield of the lignocellulosic fibers than phosphorus in the bulk. This could be due to the fact that, in the former case, the phosphorylation step required to favor dehydration occurred during fiber treatment. It could also be related to a greater proximity between phosphorus and flax.

\section{PBS/grafted flax fiber biocomposites}

When phosphorus is covalently bonded to flax for biocomposite samples, the degradation occurs in two steps whatever the treatment. The first one observed between 280 and $380{ }^{\circ} \mathrm{C}$ is relative to the pretreated or grafted flax fibers and the second one around $400{ }^{\circ} \mathrm{C}$ is relative to the degradation of the PBS matrix (Fig. 8, Table 3). As shown in Fig. 4, the variation of the first decomposition step temperature follows exactly the same trend as for grafted fibers. Moreover, the residues of biocomposites at $600^{\circ} \mathrm{C}$ increase with grafting rate and are roughly those expected from the mixing rule. In addition, it must be noted that the second peak is not (or is poorly) impacted by the presence of grafted fibers indicating that the degradation of fibers or phosphorus compounds does not interfere with the PBS decomposition. In a previous paper, ${ }^{6}$ we observed that the presence of APP, used as an additive in PBS, induced a decrease of the thermal stability of this matrix due to hot hydrolysis. Thus, the present results point out the advantage provided by the grafting strategy compared with the additive one.

Figure 5 exhibits the variation of EHC as a function of the amount of phosphorus grafted onto fibers for biocomposites containing fibers modified with DAP or P $\left(\mathrm{MAPC}_{1}(\mathrm{OH})_{2}\right)$. EHC of the biocomposites (empty symbols) decreases only slightly, while a significant difference in behavior was observed for grafted fibers (full symbols). It can be supposed that the differences between molecular and macromolecular grafting agents in biocomposites are reduced because of the diluting effect due to the presence of $70 \mathrm{wt} \%$ of PBS.

The cone calorimeter tests (Fig. 9 and Table 4) show lower (or quasi equal) values of $\mathrm{TT}$ for biocomposites containing grafted fibers (with DAP, P(MAPC $\left.(\mathrm{OH})_{2}\right)$ and P(MMA-Co-MAPC $\left(\mathrm{OH}_{2}\right)$ ) than for PBS+Tfl. This is consistent with the loss of thermal stability highlighted by TGA (Fig. 8 and Table 3 ) and PCFC (Table 3). The pHRR is always obtained just after ignition and a plateau can be observed between 150 and $400 \mathrm{~s}$ in the HRR curves, except at low phosphorus content. This type of shape is the result of the formation of a thick charring layer, limiting pyrolysis gas transfer from the sample to the flame. ${ }^{28}$ This insulating barrier layer leads to a significant decrease of the pHRR and MARHE values for the two types of fire retardant agents (up to $27 \%$ for pHRR and $29 \%$ for MARHE).

As shown previously, decomposition residues of biocomposites at high temperature (obtained by TGA) are higher for DAP treatment than for $\mathrm{P}\left(\mathrm{MAPC}_{1}(\mathrm{OH})_{2}\right)$ (or $\mathrm{P}\left(\mathrm{MMA}-\mathrm{CO}-\mathrm{MAPC}_{1}(\mathrm{OH})_{2}\right)$ ). In the preceding section, these results were assigned to a more efficient ability of phosphorus to promote char when covalently bonded to flax compared with phosphorus from the polymer side 

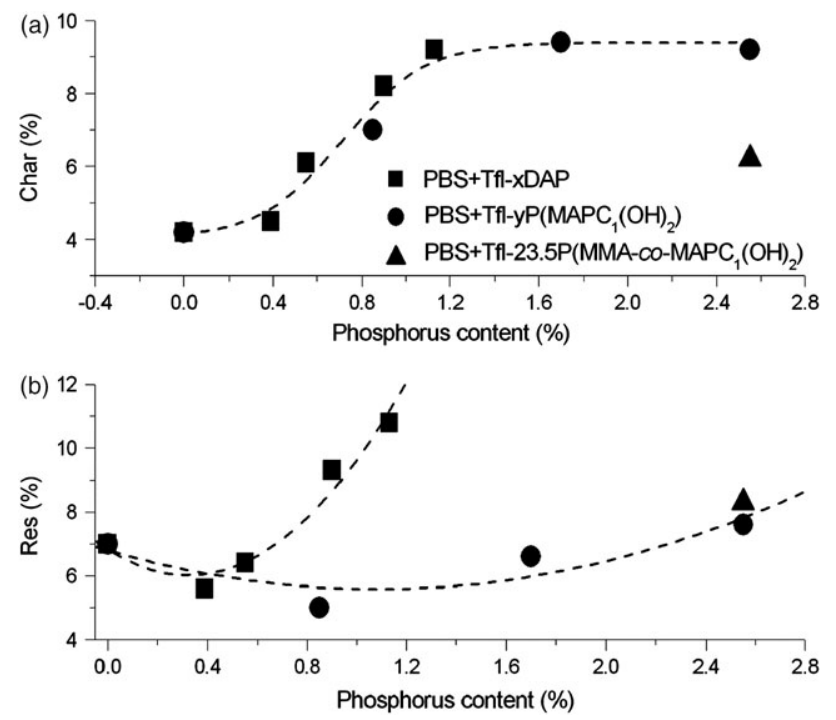

Figure 10. Comparison of (a) the char obtained after the cone calorimeter test and (b) the TGA residue.

chain. However, TGA residues are not well correlated to those of cone calorimeter tests since a significant char amount is obtained whatever the grafting agent (Fig. 10). Moreover, this char amount (obtained by the cone test) increases with the grafting rate (up to $9.2 \mathrm{wt} \%$ for the PBS+Tfl-10DAP and $9.4 \mathrm{wt} \%$ for the PBS+Tfl$\left.10 \mathrm{P}\left(\mathrm{MAPC}_{1}(\mathrm{OH})_{2}\right)\right)$. Vahabi et al. ${ }^{19}$ have highlighted the ability of $\mathrm{P}\left(\mathrm{MMA}_{\mathrm{M}} \mathrm{CO}-\mathrm{MAPC}_{1}(\mathrm{OH})_{2}\right)$ copolymers to form a residue. This behavior was attributed to the interaction of the phosphonated group of $M A P C_{1}$ with the ester group of MMA. Therefore, it can be assumed that $\mathrm{P}\left(\mathrm{MAPC}_{1}(\mathrm{OH})_{2}\right)$ or $\mathrm{P}\left(\mathrm{MMA}-\mathrm{CO}-\mathrm{MAPC}_{1}(\mathrm{OH})_{2}\right)$ also have a good ability for char formation. Hence, the global char obtained after cone calorimeter tests, in the case of phosphonated polymer grafting, could be increased due to the char acting as a sheath around the fibers and leading to a barrier effect, which limits the combustion of the biocomposite underneath its surface (Fig. 7). The residue obtained in TGA was lower with $\mathrm{P}\left(\mathrm{MAPC}_{1}(\mathrm{OH})_{2}\right)$ because the barrier effect is not significant in TGA analysis due to the low quantity of material tested. The MARHE evolution as a function of phosphorus content (Fig. 11) reinforced the hypothesis of the formation of a barrier effect. Only one behavior is observed for the MARHE which decreases with the increasing grafting rate whatever the grafting agent.

\section{CONCLUSIONS}

In this study, two types of phosphorus compounds have been grafted onto flax fibers for the enhancement of the fire behavior of biocomposites: a molecule (DAP) and two phosphonated polymers - a homopolymer $\mathrm{P}\left(\mathrm{MAPC}_{1}(\mathrm{OH})_{2}\right)$ and a copolymer $\mathrm{P}(\mathrm{MMA}-\mathrm{CO}-$ $\left.M_{A P C}(\mathrm{OH})_{2}\right)$. The TGA and PCFC tests showed a decrease of the thermal stability for the grafted fibers alone and for the fibers incorporated in the PBS matrix. This loss of thermal stability has been ascribed to the phosphorylation of flax that leads to dehydration and improvement of the amount of residue. The char residue was evidenced to be higher with DAP than with the two polymers. This effect was attributed to the higher ability of phosphorus to promote char when already covalently bonded to the lignocellulosic fibers. However, no interaction was noticed between the decomposition of natural fiber and the PBS matrix.

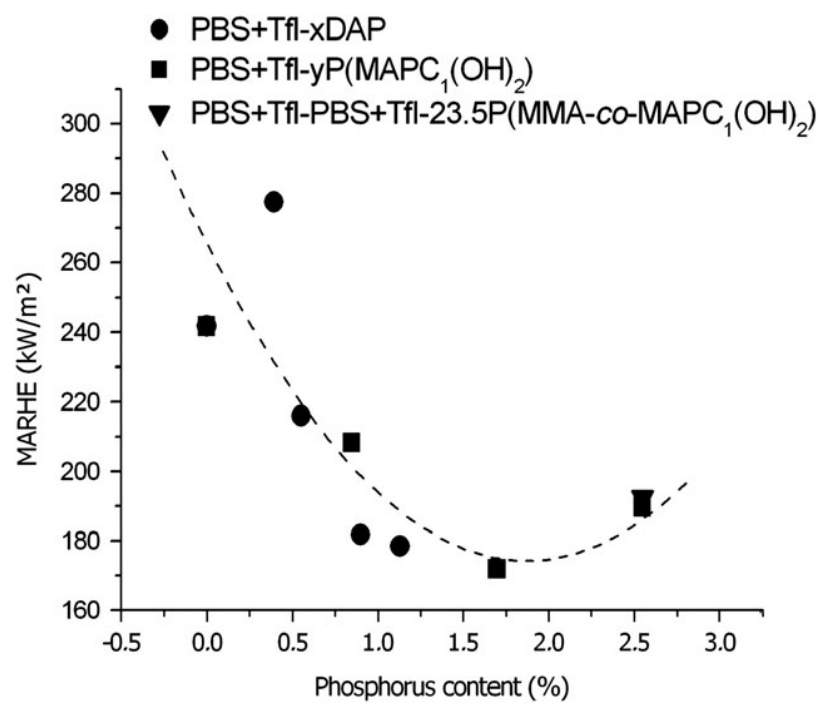

Figure 11. MARHE as a function of phosphorus content for the PBS+FTf biocomposites.

At bench scale, the cone calorimeter tests on biocomposites containing grafted fibers highlight the improvement of the fire behavior with a significant decrease of pHRR and MAHRE whatever the grafting agent. The formation of a sheath around the fibers due to the charring ability of the phosphonated polymers allows the creation of a barrier effect, and counterbalance the lack of phosphorylation. It can be expected that this kind of grafting could also improve mechanical properties. The $\mathrm{MAPC}_{1}$ monomer is easily copolymerizable with other monomers than MMA. This copolymer could play the role of a coupling agent with the matrix.

\section{REFERENCES}

1 Arbelaiz A, Fernández G, Cantero G, Llano-Ponte R and Valea A, Compos Appl Sci Manuf 36:1637-1644 (2005).

2 Belgacem MN and Gandini A, Compos Interface 12:44-75 (2005).

3 Gassan J and Bledzki A, Polym Comp 18:179-184 (1997).

4 Bledzki AK and Gassan J, Prog Polym Sci 24:221 - 274 (1999).

5 Chapple S and Anandjiwala R, J Thermoplast Compos 23:871-893 (2010).

6 Dorez G, Taguet A, Ferry L and Lopez-Cuesta JM, Polym Degrad Stab 98:87-95 (2012).

7 Kozlowski R and W ladyka-Przybylak M, Polym Advan Technol 19:446-453 (2008)

8 Gaan S and Sun G, Polym Degrad Stab 92:968-974 (2007).

9 Horrocks AR, Kandola BK, Davies PJ, Zhang S and Padbury SA, Polym Degrad Stab 88:3-12 (2005).

10 Hall ME, Horrocks AR and Seddon H, Polym Degrad Stab 64:505-510 (1998).

11 Horrocks AR and Zhang S, Polymer 42:8025-8033 (2001).

12 Shumao L, Jie R, Hua Y, Tao Y and Weizhong Y, Polym Int 59:242-248 (2009).

13 Chen D, Li J and Ren J, Polym Int 60:599-606(2011).

14 Suardana NPG, Ku MS and Lim JK, Mater Des 32:1990-1999 (2011).

15 Dorez G, Taguet A, Ferry L and Lopez-Cuesta JM, Improving the behaviour of biocomposites by fiber surface modification, in 24th Annual Conference on Recent Advances in Flame Retardancy of Polymeric Materials, Stamford, CT, 20-22 May 2013.

16 Dorez G, Otazaghine B, Taguet A, Ferry L and Lopez-Cuesta JM, Anal Appl Pyrol 105:122-130 (2014).

17 McKenna C, Higa M, Cheung N and McKenna M-C, Tetrahedron Lett 18:155-158 (1977).

18 Pothayee N, Balasubramaniam S, Davis RM, Riffle JS, Carroll MRJ, Woodward RC et al., Polymer 52:1356-1366 (2011).

19 Vahabi H, Ferry L, Longuet C, Sonnier R, Negrell-Guirao C, David G et al., Eur Polym J 48:604-612 (2012). 
20 Baley C, Brsunet F, Grohens Y and Sire O, Compos Appl Sci Manuf 37:1626-1637 (2006).

21 Lyon R and Walters RN, J Anal Appl Pyrol 71:27-46 (2004).

22 Didane N, Giraud S and Devaux E, Polym Degrad Stab 97:1083-1089 (2012).

23 Manfredi LB, Rodríguez ES, Wladyka-Przybylak Mand Vázquez A, Polym Degrad Stab 91:255-261 (2006).

24 Shen DK and Gu S, Biores Technol 100:6496-6504 (2009).
25 Soares S, Caminot G and Levchik S, Polym Degrad Stab 49:275-283 (1995).

26 Lewin M and Weil ED. Mechanisms and modes of actions, in Flame Retardancy of Polymers, in Fire Retardant Materials, ed. by Horrocks AR and Price D. CRC Press, Boca Raton, FL (2001).

27 Lecoeurs E, Vroman I, Bourbigot S, Lam TM and Delobel R, Polym Degrad Stab 74:487-492 (2001).

28 Schartel B and Hull TR, Fire Mater 31:327-354 (2007). 